\title{
Universiteit
}

Leiden

The Netherlands

\section{High finesse opto-mechanical cavity with a movable thirty-micron-size} mirror

Kleckner, D.; Marshall, W.; Dood, M.J.A. de; Dinyari, K.N.; Pors, J.B.; Irvine, W.T.M.;

Bouwmeester, D.

\section{Citation}

Kleckner, D., Marshall, W., Dood, M. J. A. de, Dinyari, K. N., Pors, J. B., Irvine, W. T. M., \& Bouwmeester, D. (2006). High finesse opto-mechanical cavity with a movable thirty-micronsize mirror. Physical Review Letters, 96(17), 173901. doi:10.1103/PhysRevLett.96.173901

Version: $\quad$ Not Applicable (or Unknown)

License: $\quad$ Leiden University Non-exclusive license

Downloaded from: https://hdl.handle.net/1887/65506

Note: To cite this publication please use the final published version (if applicable). 


\title{
High Finesse Opto-Mechanical Cavity with a Movable Thirty-Micron-Size Mirror
}

\author{
Dustin Kleckner, William Marshall, ${ }^{*}$ Michiel J. A. de Dood, ${ }^{\dagger}$ Khodadad Nima Dinyari, ${ }^{\ddagger}$ Bart-Jan Pors, ${ }^{\dagger}$ \\ William T. M. Irvine, and Dirk Bouwmeester \\ Department of Physics, University of California, Santa Barbara, California 93106, USA
}

(Received 1 November 2005; published 2 May 2006)

\begin{abstract}
We report on the demonstration of a high finesse micro-optomechanical system and identify potential applications ranging from optical cooling to weak force detection to massive quantum superpositions. The system consists of a high quality $30 \mu \mathrm{m}$ diameter flat dielectric mirror cut from a larger substrate with a focused ion beam and attached to an atomic force microscope cantilever. Cavity ring-down measurements performed on a $25 \mathrm{~mm}$ long Fabry-Pérot cavity with the $30 \mu \mathrm{m}$ mirror at one end show an optical finesse of 2100. Numerical calculations show that the finesse is not diffraction limited and that orders of magnitude higher finesse should be possible. A mechanical quality factor of more than $10^{5}$ at pressures below $10^{-3}$ mbar is demonstrated for the cantilever with a mirror attached.
\end{abstract}

PACS numbers: 42.15.Eq, 42.50.Ct, 42.50.Xa

We demonstrate a high finesse micro-optomechanical system (HFMOMS) composed of an optical cavity with a $30 \mu \mathrm{m}$ mirror on a single crystal silicon cantilever at one end. The system operates in the regime where the radiation pressure from the optical field is strong enough to influence the mechanical dynamics of the resonator. Recently, such a optomechanical coupling was seen in an elegant experiment with microtoroidal resonators [1]. In the system presented here, coupling to the light field is achieved with a micromechanical cantilever which can in turn be easily coupled to other forces.

HFMOMS are promising for a wide range of fundamental and practical applications. An important application will be the implementation of optical feedback cooling of the micro-mechanical resonator. This can be achieved either with an external feedback loop [2], or by internally delayed forces [3]. In either case, with adequately high optical quality, the effective temperature of the cantilever's fundamental vibrational mode can be drastically reduced. This could be used to improve the signal-to-noise ratio of detection of weak impulsive forces [4].

For the detection of weak continuous forces, such as those produced by precessing electron spins in a single electron spin microscope, the smallest force one can detect is limited by the bulk temperature of the cantilever. For bulk temperature, $T \lesssim 100 \mathrm{mK}$, the cantilever temperature is limited by heating due to the readout laser [5]. Attaching a high quality mirror to the readout cantilever, as done here, would both increase the finesse of the interferometric readout and reduce absorption, drastically reducing the minimum achievable temperature.

The strong interaction between the optical field and the mechanical oscillations in HFMOMS makes the cavity behave like a nonlinear medium since the length of the cavity depends upon the intensity of the field in an analogous way to the optical length of a nonlinear material [6]. The small mass and spring constant of our system would make it an ideal test bed for ponderomotive squeezing, which has been proposed as a method to reduce noise in gravitational wave detectors [7] and may also have applications in information theory [8].

The most far reaching application of HFMOMS is the study of quantum superpositions of relatively massive $\left(\sim 10^{-12} \mathrm{~kg}\right)$ systems [9-13]. Experimentally, quantum mechanics remains untested on mass scales $\gtrsim 10^{-24} \mathrm{~kg}$; the most massive superpositions to date involve $\sim 10^{6}$ electrons in superconducting devices [14,15], and large $(\sim 100$ atom $)$ molecules in an atom interferometer $[16,17]$. Among existing proposals, one scheme [11-13] uses the radiation pressure of a single photon to create a macroscopic superposition involving two spatially distinct locations of a mirror attached to a mechanical oscillator. The technical feasibility of this scheme has been explored in [18]. Essential to this proposal is the construction of a high finesse cavity to increase the radiation pressure of a single photon to an extent where it can displace the cantilever by at least the width of a ground state wave packet. Achieving the superposition as discussed in [18] is an extraordinary challenge; it requires a cavity of $25 \mathrm{~mm}$ length with a $20 \mu \mathrm{m}$ diameter mirror on a micromechanical resonator with total mass $\sim 10^{-12} \mathrm{~kg}$, an optical finesse, $F \cong 10^{6}$, and mechanical quality, $Q \cong 10^{5}$. Furthermore, the system must operate in ultrahigh vacuum and at $\mathrm{mK}$ temperatures. With continued improvements to the optical quality of the system described here and possibly aided by optical cooling, these requirements could potentially be within reach.

We fabricated and studied an optical cavity composed of a macroscopic spherical mirror and a microscopic plane mirror on the end of an atomic force microscope (AFM) cantilever (Fig. 1). Both mirrors are distributed Bragg reflectors (DBR), with alternating layers of $\mathrm{SiO}_{2}(n=$ 1.46) and $\mathrm{Ta}_{2} \mathrm{O}_{5}(n=2.04)$. For our preliminary investigation, we used a 30 layer stack, designed to have $R>$ $99.995 \%$ at $780 \mathrm{~nm}$. This gives a reflection-limited finesse of $6 \times 10^{4}$. Thicker DBR stacks of this type have demon- 


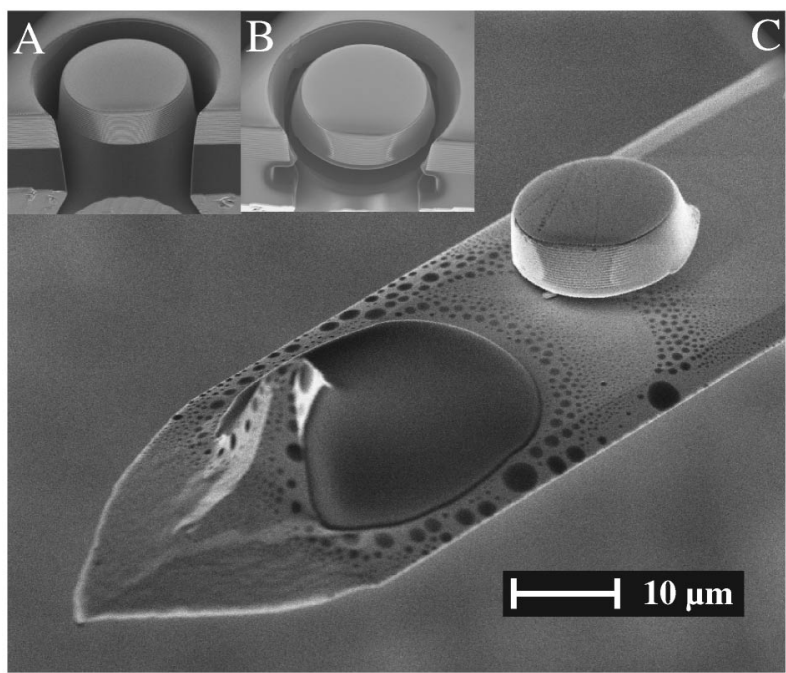

FIG. 1. Scanning electron microscopy images of a $15 \mu \mathrm{m}$ prototype mirror during the cutting process (a), (b) and after attachment to a cantilever (c). Small scratches are visible on the mirror in (c) from the attachment process. The fabrication procedure was subsequently adjusted to prevent this.

strated high enough reflectivity for finesses in excess of $10^{6}$ $[19,20]$. The large mirror has an effective diameter of $6 \mathrm{~mm}$ and a radius of curvature of $25 \mathrm{~mm}$. The microscopic mirror lies at the center of the radius of curvature of the large mirror. The small mirror used for most of the measurements has diameter $30 \mu \mathrm{m}$, although smaller mirrors were also fabricated and tested. A diagram of the experimental setup is shown in Fig. 2.

To make the small mirrors, we begin with a DBR stack deposited on a conductive silicon substrate. Using a focused ion beam (FIB), we first cut away a ring of material to define the mirror edge [Fig. 1(a)]. We then rotate the sample $96^{\circ}$ in order to cut out the bottom of the mirror [Fig. 1(b)]. The additional $6^{\circ}$ is used to compensate for the tapered edges of a FIB cut produced at high ion currents. The bottom cut is stopped before it completely frees the mirror, thus leaving it connected by $1-2 \mu \mathrm{m}$ of silicon. We then place a small drop of low-viscosity optical epoxy near the tip of an AFM cantilever using a several micron thick pulled glass rod on a manipulation arm. Next, using a fresh glass tip, we gently break the mirror free from the substrate. To avoid scratching the mirror, we use the side of the glass rod instead of the sharp tip. Once freed, the detached mirror is carefully lifted from the substrate via electrostatic interaction with the glass rod. It is then transferred to the cantilever and placed on the drop of epoxy [Fig. 1(c)].

To characterize the optical cavity, we measure its spectral response using a tunable laser diode (TLD). The transmission of a Fabry-Pérot cavity with regards to frequency, $f$, and changes in cavity length, $\Delta x$, is

$$
T \propto \frac{1}{1+\frac{4 F^{2}}{\pi^{2}} \sin ^{2}\left[\pi\left(\frac{f}{\Delta f}+\frac{2 \Delta x}{\lambda}\right)\right]},
$$

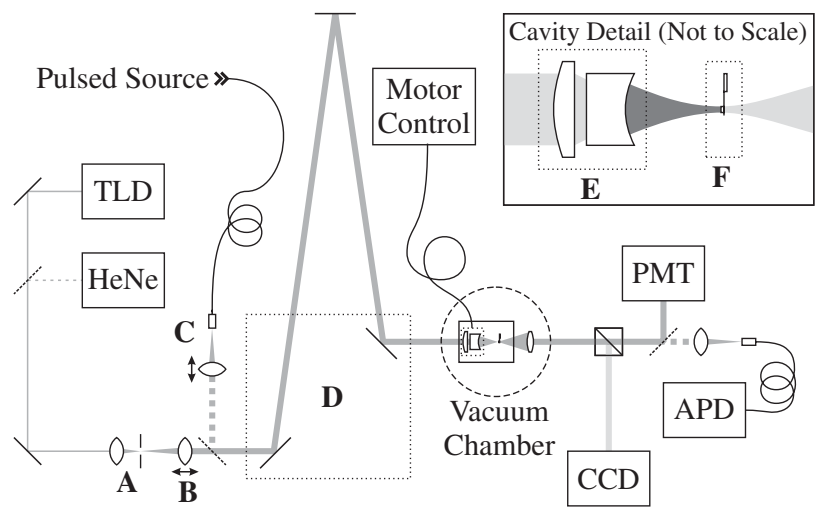

FIG. 2. Diagram of the experimental setup. A $780 \mathrm{~nm}$ TLD is used for frequency scanned measurements. A $633 \mathrm{~nm}$ helium neon laser is used for alignment. The light from either laser is then passed through a spatial filter (A) and collimated with a lens (B). The lens is chosen to match the cavity mode. For the ring-down measurements a $780 \mathrm{~nm} 200 \mathrm{fs}$ pulsed laser is coupled in via a fiber (C). A periscope (D) aligns light to the cavity. The large mirror and an incoupling lens (E) are mounted on a motorized stage allowing control of tip/tilt as well as the overall length of the cavity in vacuo. The cantilever or small mirror (F) are mounted on a Gimbal mount which is prealigned outside of the vacuum chamber. A fraction of the light leaving the cavity is used for imaging on a CCD, while the remainder is sent either to a PMT or APD.

where $\lambda$ is the laser wavelength, and $\Delta f=\frac{c}{2 L}$ is the free spectral range, which is $6 \mathrm{GHz}$ for our cavity. The proportionality factor is 1 for cavities with mirrors of equal reflectivity and less than 1 otherwise. The optical finesse, $F$, a useful measure of the cavity quality, is the ratio of peak width to free spectral range for the periodic peaks of Eq. (1). Using the TLD we sweep the frequency by slightly more than a free spectral range and monitor the cavity transmission on a photomultiplier tube (PMT) (Fig. 3). The power incident on the cavity is roughly $1 \mathrm{~mW}$. In practice a series of several reoccurring peaks is observed due to higher order modes and imperfect mode matching. The peak of the fundamental mode can easily be identified by its higher finesse and location in the spectrum. If the laser is scanned slowly enough, thermal vibrations of the cantilever at the primary mechanical resonance frequency of $12.5 \mathrm{kHz}$ are clearly visible [Fig. 3(b)]. This is because the time it takes the TLD to scan over the peak is several mechanical oscillations long. Scanning at a rate $\gtrsim 10 \mathrm{~Hz}$ reduces the effect of the vibrations and enables a measurement of linewidth of the cavity optical resonance. The maximum finesse measurable by this method is $1020 \pm$ 50 and is limited by the $6 \mathrm{MHz}$ TLD linewidth.

Cavity ring-down provides an alternative method for determining the finesse. The mean number of round trips of a photon in a Fabry-Pérot cavity is given by $\langle n\rangle=\frac{F}{2 \pi}$. The corresponding exponential decay time is $\tau=\frac{L F}{\pi c}$. To measure this decay we pumped the cavity with $200 \mathrm{fs}$ laser 


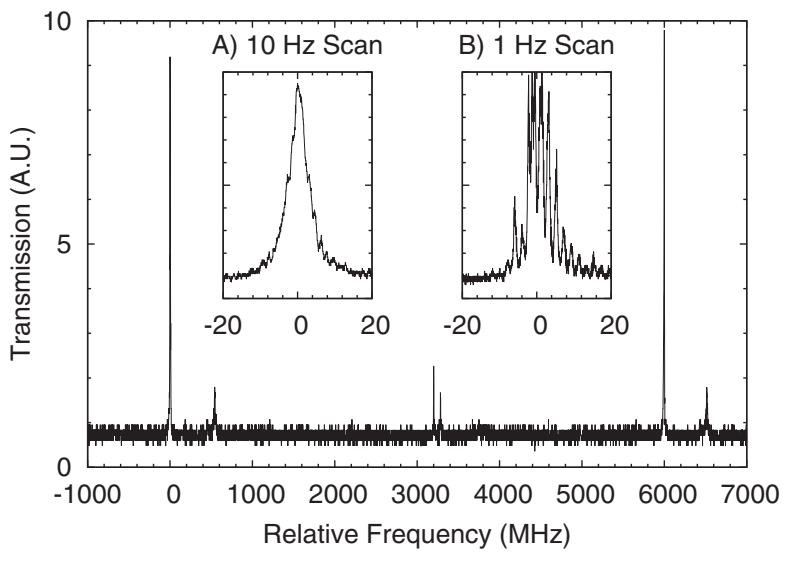

FIG. 3. Fabry-Pérot scan (peaks inset). Higher order modes are visible; adjustment of the incoupling reveals that the cavity supports several more. (a) The Lorentzian peak has FHWM $5.9 \pm 0.2 \mathrm{MHz}$, resulting in a finesse (limited by the laser linewidth) of $1020 \pm 50$. (b) If the laser is scanned at a lower rate, thermal vibrations of the cantilever become visible.

pulses at $780 \mathrm{~nm}$ with a repetition rate of $40 \mathrm{kHz}$. Light leaving the cavity was sent to an avalanche photodiode (APD) capable of detecting individual photons. The APD pulses were monitored on a multichannel scaler triggered by the laser electronics. The summed results of $10^{5}$ pulses are shown in Fig. 4. The cavity alignment was unchanged from the earlier measurements using the TLD. The measured finesse by this method is $2100 \pm 50$. The finesse could not be significantly increased beyond this point by alignment.

We now discuss limitations on the optical finesse set by the finite size of the cavity mirrors. Because of diffraction losses, the concept of eigenmodes of the cavity breaks

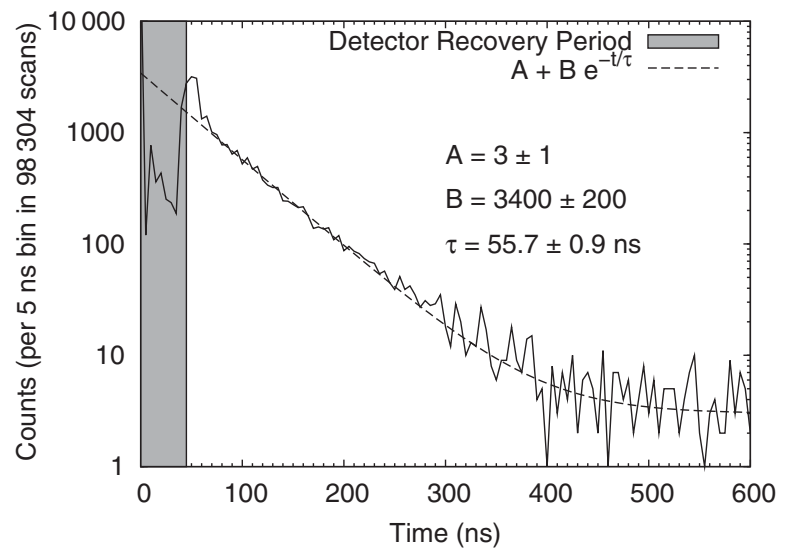

FIG. 4. Cavity ring-down measurement. A laser pulse enters the cavity at time 0 . The scattered light is bright enough to saturate the APD, resulting in a $50 \mathrm{~ns}$ dead time. The light intensity is low enough after the recovery that saturation effects can be ignored. A fit of the data from 100-2000 ns demonstrates a finesse of $2100 \pm 50$. The slightly faster decay at $50 \mathrm{~ns}$ is due to light leaking from higher order modes. down and is replaced by modes that decay at a constant rate. The calculation of these modes can be reduced to a round-trip matrix problem by expanding the optical field in the cavity in terms of the cylindrically symmetric $(m=0)$ Laguerre-Gaussian modes which are the propagation eigenmodes of the paraxial wave equation for a cavity with infinitely large mirrors. The clipping due to each mirror is represented by a mode mixing matrix whose elements are the mode overlap integrals calculated only over the surface of the mirror. The effect of one round-trip propagation is the product of the mixing matrices for each mirror. The eigenvalues and eigenvectors of this matrix correspond to the cavity modes and can be used to calculate the finesse (Table I) of each.

Many of the obtained finesses exceed those limited by realistic mirror reflectivities [20]. Since the finesse is limited by multiple independent loss mechanisms, the total finesse can be calculated from the limiting finesses for each loss mechanism considered separately: $\frac{1}{F}=$ $\frac{1}{F_{\text {diff. }}}+\frac{1}{F_{\text {refl. }}}+\cdots$. To determine the diffractive losses of single point defects in the mirror, calculations were carried out with the center $2 \mu \mathrm{m}$ of the small mirror removed. As can be seen in Table I, this results in a decrease in the overall finesse as well as a reduction in the number of efficiently propagating modes. Conversely, the number of prominent modes is not affected by a decrease in overall reflectivity. The frequency scanned measurements show that the number of prominent higher order modes for 20 and $30 \mu \mathrm{m}$ mirrors is consistent with the calculations for defectless mirrors. We thus suspect that the finesse limitation is due to scattering off of small surface contaminants, effectively reducing the reflectivity. Furthermore, we believe these contaminants are sputtered particles from the FIB cutting and we are developing methods to eliminate them.

To measure the mechanical quality of the system, we observe the thermal vibration spectrum of the cantilever. This is done by locking the frequency of the TLD to the side of a cavity transmission peak with a slow $(10 \mathrm{~Hz})$ feedback loop. The thermal vibrations of the cantilever, with an rms amplitude of $1.5 \AA$, are immediately visible as fluctuations in the output intensity. The finesse is inten-

TABLE I. Maximum optical finesse for finite-sized mirror cavities of the type presented here $(\lambda=780 \mathrm{~nm})$.

\begin{tabular}{lcc}
\hline \hline \multicolumn{1}{c}{ Mirror Sizes } & $\begin{array}{c}\text { Primary Mode } \\
\text { Finesse }\end{array}$ & $\begin{array}{c}\text { Higher Mode } \\
\text { Finesses with } F>10\end{array}$ \\
\hline $6 \mathrm{~mm}, 15 \mu \mathrm{m}$ & $3.9 \times 10^{4}$ & 83 \\
$6 \mathrm{~mm}, 20 \mu \mathrm{m}$ & $3.5 \times 10^{6}$ & 3500,24 \\
With $2 \mu \mathrm{m} \mathrm{defect}$ & 6000 & 39 \\
$6 \mathrm{~mm}, 30 \mu \mathrm{m}$ & $3.6 \times 10^{10}$ & $1.4 \times 10^{7}, 2.7 \times 10^{4}, 190$ \\
With $2 \mu \mathrm{m} \mathrm{defect}$ & $4.5 \times 10^{5}$ & $3.0 \times 10^{4}, 240,13$ \\
$8 \mathrm{~mm}, 20 \mu \mathrm{m}$ & $1.6 \times 10^{9}$ & $8.0 \times 10^{5}, 2200,24$ \\
\hline \hline
\end{tabular}


TABLE II. $Q$ of the cantilever at different pressures.

\begin{tabular}{lc}
\hline \hline \multicolumn{1}{c}{ Pressure } & Mechanical Quality Factor \\
\hline 1 Atm. & $79.7 \pm 0.3$ \\
$1 \mathrm{mbar}$ & $1479 \pm 8$ \\
$5 \times 10^{-3} \mathrm{mbar}$ & $(7.91 \pm 0.08) \times 10^{4}$ \\
$1 \times 10^{-4} \mathrm{mbar}$ & $(1.37 \pm 0.03) \times 10^{5}$ \\
\hline \hline
\end{tabular}

tionally reduced to 150 by slight cavity misalignment to prevent transient vibrations from unlocking the feedback loop. By monitoring the transmission fluctuations on a spectrum analyzer, we can determine the spectral width of the fundamental cantilever vibrational mode, centered at $12.5 \mathrm{kHz}$. The mechanical quality factor is then given by $Q=\frac{\omega_{0}}{\Delta \omega_{\mathrm{FWHM}}}$.

For micromechanical systems, $Q$ is a strong function of pressure. At atmospheric pressure, viscous damping typically dominates $Q$. As the pressure is decreased, $Q$ increases until it becomes intrinsically limited by cantilever material or construction. As expected, this linewidth narrowing is observed as the pressure is reduced (Table II). An intrinsically limited $Q$ of $\sim 10^{5}$ is seen at pressures below $10^{-3}$ mbar. This is consistent with the expected value for our cantilever dimensions $(450 \times 50 \times 2 \mu \mathrm{m})$ [21]. It appears therefore that the mechanical $Q$ of the cantilever is not affected by the mirror attachment process.

Our system already shows considerable promise for optical cooling; the thermal vibrations of the cantilever are currently visible with a signal-to-noise ratio $\gtrsim 10^{5}$, implying that optical feedback cooling [2] of the center-ofmass motion of the cantilever to $<1 \mathrm{~K}$ is possible from room temperature.

It should be possible to observe nonlinear effects due to optomechanical coupling with the current system by modest increases in either finesse or input power. Alternatively, if the reflectivity of the larger mirror in our current cavity were reduced to match the effective reflectivity of the tiny mirror, nonlinear effects would become significant with input powers of order $100 \mu \mathrm{W}$ [7].

Concerning further improvement of our system, we have shown numerically that a finesse several orders of magnitude higher should be possible with improved mirror fabrication techniques. In order to use the system to realize a macroscopic superposition, it also needs to be shown that mirrors can be attached to significantly thinner and lighter cantilevers and that high mechanical quality factors can be maintained in such cases. If this can be achieved, the most significant barriers to creating a massive "Schrödinger's cat" state, as proposed in [18], will have been overcome.

The authors would like to acknowledge D. Cannell, C. Simon, S. Hastings-Simon, A. Kohl, and G. Khoury for useful discussions and experimental support. This work was supported through NSF Grants No. 0304678 and No. 0504825. We thank Nano Devices Inc., Santa
Barbara for the donation of test cantilevers and R. Lalezari of Advanced Thin Films for the DBR sputter deposition run and advice.

*Present address: Belfer Center for Science and International Affairs, Harvard University, 79 JFK St., Cambridge, MA 02138, USA.

†Present address: Huygen's Laboratory, Leiden University, P.O. Box 9504, 2300 RA, The Netherlands.

${ }^{*}$ Present address: Department of Physics, University of Oregon, 1371 E. 13th Ave., Eugene, OR 97403, USA.

[1] H. Hokhsari, T.J. Kippenberg, T. Carmon, and K.J. Vahala, Opt. Express 13, 5293 (2005).

[2] M. Pinard, P. F. Cohadon, T. Briant, and A. Heidmann, Phys. Rev. A 63, 013808 (2001).

[3] C. H. Metzger and K. Karrai, Nature (London) 432, 1002 (2004).

[4] D. Vitali, S. Mancini, and P. Tombesi, Phys. Rev. A 64, 051401(R) (2001).

[5] H. J. Mamin and D. Rugar, Appl. Phys. Lett. 79, 3358 (2001).

[6] S. Mancini and P. Tombesi, Phys. Rev. A 49, 4055 (1994).

[7] T. Corbitt, Y. Chen, F. Khalili, and D. Ottaway, Phys. Rev. A 73, 023801 (2006).

[8] S. Giannini, S. Mancini, and P. Tombesi, Quantum Inf. Comput. 3, 265 (2003).

[9] J. Ruostekoski, M. J. Collett, R. Graham, and D. F. Walls, Phys. Rev. A 57, 511 (1998).

[10] J. I. Cirac, M. Lewenstein, K. Molmer, and P. Zoller, Phys. Rev. A 57, 1208 (1998).

[11] D. Bouwmeester, J. Schmiedmayer, H. Weinfurter, and A. Zeilinger, in Gravitation and Relativity: At the Turn of the Millenium, edited by N. Dadhich and J. Narliker (IUCAA, Pune, 1998).

[12] E. R. Penrose, in Wavefunction Collapse as a Real Gravitational Effect, edited by A.S. Fokas, A. Grigoryan, T. Kibble, and B. Zegarlinski, Mathematical Physics 2000 (Imperial College, London, 2000).

[13] S. Bose, K. Jacobs, and P. L. Knight, Phys. Rev. A 59, 3204 (1999).

[14] C. H. van der Wal, A. C. J. ter Haar, F. K. Wilhelm, R. N. Schouten, C. J. P. M. Harmans, T. P. Orlando, S. Lloyd, and J. E. Mooij, Science 290, 773 (2000).

[15] J. Friedman, V. Patel, W. Chen, S. Tolpygo, and J. Lukens, Nature (London) 406, 43 (2000).

[16] M. Arndt, O. Nairz, J. Vos-Andreae, C. Keller, G. van der Zouw, and A. Zeilinger, Nature (London) 401, 680 (1999).

[17] W. Schöllkopf and J.P. Toennies, Science 266, 1345 (1994).

[18] W. Marshall, C. Simon, R. Penrose, and D. Bouwmeester, Phys. Rev. Lett. 91, 130401 (2003).

[19] C. J. Hood, H.J. Kimble, and J. Ye, Phys. Rev. A 64, 033804 (2001).

[20] G. Rempe, R. Thompson, H. Kimble, and R. Lalezari, Opt. Lett. 17, 363 (1992).

[21] F. R. Blom, S. Bouwstra, M. Elwenspoek, and J. H. J. Fluitman, J. Vac. Sci. Technol. B 10, 19 (1992). 